\title{
Cáncer de mama en mujeres adultas mayores, características clínicas e histopatológicas y resultados del tratamiento con intención curativa
}

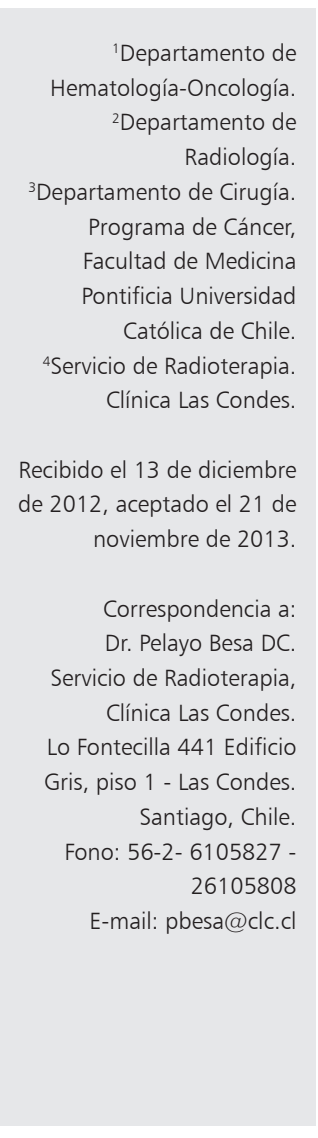

\author{
CESAR SÁNCHEZ R. ${ }^{1}$, FEDERICO BAKAL I. ${ }^{2}$, \\ MAURICIO CAMUS A. ${ }^{3}$, PELAYO BESA DE C. ${ }^{4}$
}

\section{Characteristics and results of breast cancer in elderly females treated with curative intent}

Background: Aging is the main risk factor to acquire breast cancer (BC). Nevertheless $B C$ in elderly patients is sub-represented in clinical trials. Aim: To describe the clinical characteristics and long term treatment results of localized BC in older women. Material and Methods: Review of medical records of 65 women aged 70 to 88 years, with localized BC, treated with surgery, postoperative radiotherapy or systemic therapy at a Clinical Hospital in Chile. Results: The presence of symptoms or abnormal findings on physical examination were the main reasons for consulting in $65 \%$ of cases. Compared with tumors detected on physical examination, those detected using screening mammogram were smaller and were in stage 1 with a higher frequency (18 and 59\% respectively, $p<0.01$ ). The pathological type was luminal in $80 \%$ of cases. Overall survival was better for luminal pathological type. All patients completed radiotherapy without interruptions developing minor acute toxicity. The most common co-morbidity was high blood pressure occurring in $46 \%$ of patients. Thirteen percent of patients had three or more co-morbidities. After a median follow up of 7 years, 23 (35\%) patients had died and the cause of death was BC in 43\% of cases. Two patients died of lung cancer. No patient had a local breast relapse. Conclusions: Screening mammogram in older women detected smaller tumors and it was associated with a better survival. BC is the ultimate cause of death in approximately half of cases.

(Rev Med Chile 2013; 141: 1534-1540)

Key words: Adverse effects; Aged; Breast neoplasms; Carcinoma, ductal, breast.

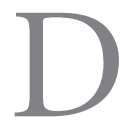
ebido al aumento en las expectativas de vida, se estima que en el año 2025 existirán en Chile 110 adultos mayores (AM) de 60 años por cada 100 menores de 15 años $^{1,2}$. Siendo el cáncer una patología asociada al envejecimiento ${ }^{3}$, este cambio demográfico implica que la incidencia de neoplasias epiteliales aumentará en las próximas décadas.

El cáncer de mama $(\mathrm{CM})$ es la primera causa de muerte asociada a neoplasias en mujeres chilenas $^{4}$, y la edad, el principal factor de riesgo para su desarrollo ${ }^{3}$.
Los cambios biológicos y clínicos producidos por el envejecimiento alteran la historia natural y tratamiento del cáncer ${ }^{5-7}$. Sin embargo, menos del $10 \%$ de los pacientes ingresados a ensayos clínicos fase III son mayores de 70 años ${ }^{8}$. La mayoría de las publicaciones sobre indicaciones de tratamiento $y$ prevención de cáncer en AM con CM son derivadas de la experiencia con pacientes más jóvenes ${ }^{3,5}$; siendo muy interesante desarrollar estudios dirigidos específicamente a esta población.

El objetivo de este trabajo es caracterizar y evaluar resultados de tratamiento en pacientes 
Cáncer de mama en mujeres adultas mayores - C. Sánchez et al

mayores de 70 años con CM, no metastásico, tratados con intención curativa en nuestro centro.

\section{Pacientes y Métodos}

Se realizó un estudio descriptivo, retrospectivo. Se analizaron las historias clínicas de 65 pacientes de 70 o más años con diagnóstico de CM infiltrante no metastásico, etapas I a IIIB (AJCC 6 a edición) $^{9}$, tratadas con intención curativa entre enero del año 1997 y mayo del año 2004 en el Centro de Cáncer de la Pontificia Universidad Católica de Chile. Se registró: edad, motivo de consulta (hallazgos mamográficos o por palpación), co-morbilidades, hallazgos patológicos del tumor y de linfonodos axilares y tratamiento sistémico (tamoxifeno y/o quimioterapia).

El análisis patológico incluyó: tamaño tumoral, tipo histológico, grado de diferenciación según la clasificación de Elston y Bloom, modificada por Elston y Ellis ${ }^{10,11}$, estudio de receptores de estrógeno (RE) y progesterona (RP), escala de $0-100 \%$, HER 2 (Receptor de factor de crecimiento epidérmico tipo 2) escala de $+\mathrm{a}+++$. Todos por métodos de inmuno-histoquímica. Siendo para HER2: + negativo, ++ incierto evaluado adicionalmente por FISH y +++ positivo. Se definió el subtipo tumoral clínico-patológico según los siguientes elementos ${ }^{12-14}$ : Luminal A ( RE + y/o RP +, grado histológico (GH) 1-2, HER2 negativo); Luminal B ( RE + y/o RP +, GH 3 y /o HER 2 positivo), Triple negativo (RE -, RP -, y HER negativo), HER2+ (RE - y RP -, HER 2 positivo). No se cuenta con Ki67, que mide el grado de proliferación celular, en el período del estudio. En pacientes con más de un tumor en la mama, se reporta el tumor de mayor tamaño ${ }^{15}$.

El tratamiento se inició con la mastectomía y disección axilar. La mastectomía fue parcial o total dependiendo de las condiciones anatómicas, el tamaño tumoral y la preferencia de la paciente ${ }^{16,17}$. Luego, dependiendo de si se realizó tratamiento conservador (mastectomía parcial) y del estadio patológico, definido como la etapa del tumor de acuerdo a la clasificación TNM (T: tamaño del tumor primario, N: número de linfonodos comprometidos con tumor y $\mathrm{M}$ : presencia o ausencia de metástasis) $)^{9,15}$, se administró con acelerador lineal Varian 2300 CD y 2100 EX, radioterapia a la mama o a la pared torácica y regiones linfáticas, recibiendo una dosis de 50 Gy en 25 fracciones $^{16,17}$.
Se adicionó una sobreimpresión de 10 Gy al lecho tumoral o cicatriz de mastectomía según decisión del tratante ${ }^{16,17}$. Se evaluó semanalmente la toxicidad aguda durante la radioterapia ${ }^{18}$. Finalmente, se indicó tratamiento adyuvante sistémico según decisión del tratante $e^{16,17}$.

Evaluamos el resultado de los tratamientos con un seguimiento hasta octubre del año 2011: recurrencias loco-regionales $(\mathrm{RL})$, recurrencias a distancia (RD), sobrevida libre de enfermedad (SLE), sobrevida global (SG) y sobrevida específica de enfermedad (SEE). Se definió RL al desarrollo de cáncer confirmado por biopsia en áreas irradiadas ${ }^{19}$. La RD se definió como la aparición de metástasis de adenocarcinoma de posible origen mamario fuera del campo de radiación, confirmadas histológicamente ${ }^{19}$, exceptuando la mama contralateral, que se consideró como segundo primario, un nuevo $\mathrm{CM}^{19}$. La SG se definió como el tiempo transcurrido desde la mastectomía hasta el fallecimiento por cualquier causa. La fecha y causas de muerte se obtuvieron de datos de los certificados de defunción y de la historia clínica. Para la SEE se definió el mismo período que la SG y se contabilizó sólo las pacientes que fallecen de CM. La SLE se definió como el tiempo transcurrido desde la mastectomía hasta la confirmación histológica de una recurrencia ${ }^{19}$, loco-regional o a distancia, la aparición de un segundo primario o la muerte por cualquier causa. El seguimiento se realizó periódicamente, cada 3 meses el primer año, cada 4 el segundo año, cada 6 desde el tercer al quinto año y luego anualmente. Las pacientes que no asistieron a control por más de un año fueron entrevistadas telefónicamente. El seguimiento se realizó hasta la fecha de muerte.

Estadística: Se utilizó el test de $\chi^{2}$, para comparar las variables discretas. El método de Kaplan-Meier se utilizó para evaluar las curvas de sobrevida. La comparación de los resultados de sobrevida se realizó con el test de Log-Rank. Todos los valores reportados de $\mathrm{P}$ son de dos colas, con significancia estadística significativa con valores menores o iguales a 0,05 . Se utilizó programa estadístico SPSS versión 19.0.

\section{Resultados}

\section{Pacientes y características tumorales:}

Las características de las pacientes se resumen en la Tabla 1. La edad promedio de las pacientes 
Tabla 1. Características de pacientes

\begin{tabular}{|lcc|}
\hline Características & n & \% \\
\hline Total & 65 & 100 \\
\hline Edad & & \\
$\quad 70-79$ años & 48 & 74 \\
80 - 89 años & 17 & 26 \\
n de Co-morbilidades & & \\
0 & 16 & 25 \\
1 & 21 & 32 \\
2 & 19 & 29 \\
3 o más & 9 & 14 \\
Motivo de consulta & & \\
$\quad$ Mamografía & 22 & 33 \\
Clínica & 42 & 65 \\
Sin datos & 1 & 2 \\
\hline
\end{tabular}

fue 76,8 años (70-88) y $26 \%$ eran mayores de 80 años. Setenta y cuatro por ciento de las pacientes era portadora de co-morbilidades al momento de diagnosticarse el CM y $42 \%$ tenía dos o más co-morbilidades, siendo la más frecuente hipertensión arterial $(30 \%)^{20}$.

El motivo de consulta fue sospecha de cáncer en la mamografía de control preventivo ${ }^{16,17}$, tamizaje, en 22 pacientes (34\%). De las restantes pacientes, $42(65 \%)$ consultaron por una masa palpable y en un caso no se obtuvo el dato. La estadificación patológica del $\mathrm{CM}^{9}$ correspondió a etapa I en $32 \%$, etapa II en $45 \%$ y etapa III en $23 \%$. En 3 pacientes se estadificó sólo con la información clínica ${ }^{9}$. Las pacientes que efectuaron control preventivo con mamografía, detectaron el CM en etapa I en 59\% de los casos. En cambio sólo 18\% de las mujeres detectaron por palpación el CM en etapa I $(p=0,0055)$.

Las características patológicas del CM se resumen en la Tabla 2. El tamaño promedio del tumor mamario infiltrante fue de 21,3 $\mathrm{mm}$ y $91 \%$ tenían 5 o menos $\mathrm{cm}$ de diámetro mayor. Los márgenes quirúrgicos fueron negativos, definidos como mayor o igual a $1 \mathrm{~mm}$, en $94 \%$ de los casos ${ }^{21}$. El tipo histológico más frecuente, $72 \%$, fue el carcinoma ductal infiltrante, luego el carcinoma lobulillar con $14 \%$ y el resto correspondió a otras variantes histológicas: tubular, metaplásico, etc. El GH se determinó en $77 \%$ de los casos y correspondió a GH I en $16 \%$, grado II en $68 \%$ y grado III en $16 \%$. La determinación de receptores de estrógeno
Tabla 2. Características tumorales

\begin{tabular}{|c|c|c|}
\hline & $\mathbf{n}$ & $\%$ \\
\hline Total tumores & 69 & 100 \\
\hline \multicolumn{3}{|l|}{ Histología } \\
\hline Ductal & 50 & 72 \\
\hline Lobulillar & 10 & 15 \\
\hline Otro & 9 & 13 \\
\hline \multicolumn{3}{|l|}{ Tamaño tumoral } \\
\hline $0-2 \mathrm{~cm}$ & 31 & 45 \\
\hline $2,1-5 \mathrm{~cm}$ & 29 & 42 \\
\hline Más de $5 \mathrm{~cm}$ & 6 & 9 \\
\hline Sin info & 3 & 4 \\
\hline \multicolumn{3}{|l|}{ Linfonodos } \\
\hline Negativos & 32 & 49 \\
\hline Positivos & 27 & 42 \\
\hline Sin info & 6 & 9 \\
\hline \multicolumn{3}{|l|}{ Etapa } \\
\hline I & 21 & 32 \\
\hline$\|$ & 29 & 45 \\
\hline III & 15 & 33 \\
\hline \multicolumn{3}{|c|}{ Clasificación clínico patológico } \\
\hline Luminal A & 26 & 40 \\
\hline Luminal B & 14 & 21 \\
\hline Triple negativo & 5 & 8 \\
\hline Her2 & 3 & 5 \\
\hline No clasificable & 17 & 26 \\
\hline
\end{tabular}

*SIN INFO: Sin información patológica completa. Se utilizó la etapificación clínica.

y progesterona fue positiva en la mayoría de los tumores (91\%), los que hoy se clasifican como tumores luminales. La expresión de RE tenía una intensidad mayor a $80 \%$ en $67 \%$ de los casos (mediana 95\%, rango: 30-100).

Se realizó estudio de HER2 por método inmuno-histoquímico en $83 \%$ de los casos y fueron positivos para el marcador $13 \%$.

Se clasificó el subtipo tumoral clínico-patológico de los tumores correspondiendo a un patrón luminal A, $40 \%$ de los casos, luminal B 22\%, triple negativo $8 \%$ y HER2 positivo $5 \%$. No se pudo clasificar el subtipo tumoral en $25 \%$ de los casos por ausencia del análisis de HER2 y/o del GH. El estudio patológico demostró metástasis nodal axilar en $42 \%$ de los casos. No se realizó disección axilar en 6 pacientes con axila clínicamente negativa. No disponemos de información acerca de la causa de esta última decisión. 


\section{Patrón de tratamiento}

Se trataron con mastectomía parcial 53 pacientes, de ellos 28 con disección axilar (52,7\%), y 12 con mastectomía radical modificada. Las 65 pacientes fueron tratadas con radioterapia post operatoria $^{16,17}$. Recibieron tratamiento médico adyuvante con tamoxifeno $65 \%$ de las pacientes y con quimioterapia 9\%. No se administró terapia sistémica a $26 \%$ de las pacientes. Ochenta por ciento de pacientes clasificadas con subtipo tumoral luminal recibieron tamoxifeno.

\section{Sobrevida}

La mediana de seguimiento fue de 6,9 años (0,5-12,3 años). Ha fallecido $35 \%$ de las pacientes $y$

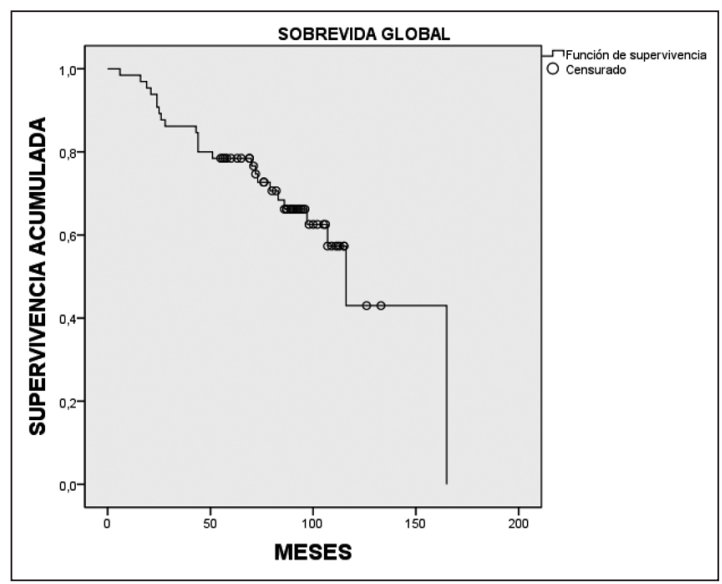

Figura 1. Sobrevida global de pacientes adultos mayores con cáncer de mama.

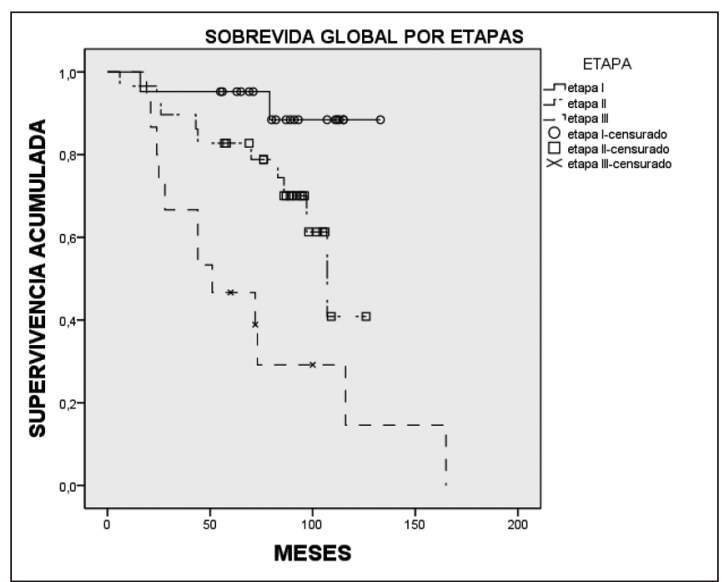

Figura 3. Sobrevida global según etapas. la causa ha sido CM en $43 \%$ de los casos (Figura 1). La mediana de SG fue 116 meses (99-133 meses). En el análisis univariado, las pacientes diagnosticadas por mamografía preventiva tuvieron una SG más prolongada que las diagnosticadas por palpación tumoral (Log Rank p: 0,007) (Figura 2). La mediana de sobrevida para pacientes en etapa I no se ha alcanzado, en etapa II fue 107 meses (IC95: 89-125) y en etapa III: 51 meses (IC95: 11-91); diferencias estadísticamente significativas ( $\log$ Rank p: 0,002) (Figura 3). La diferencia en SG no fue significativa para los grupos etarios de 70-79 años, comparado con las de 80 o más años ( $\log$ Rank p: 0,75) (Figura 4). Para las co-morbilidades 0-1 comparado con 2 o más (Log Rank p: 0,55)

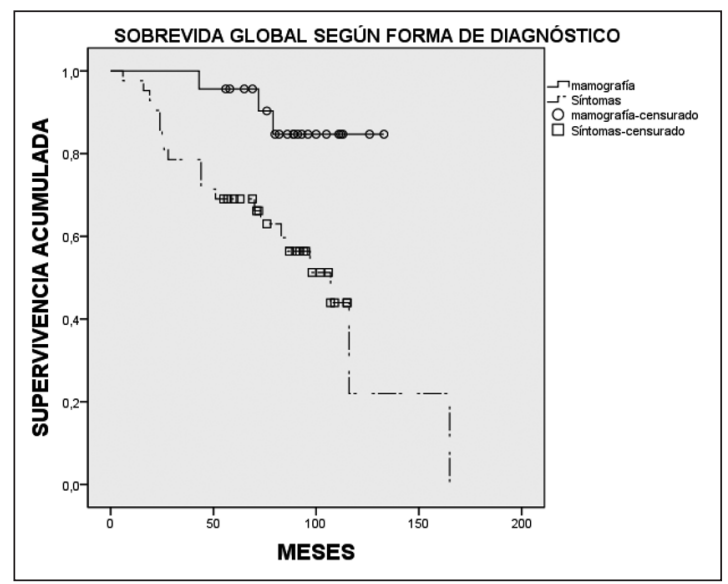

Figura 2. Sobrevida global según forma de diagnóstico.

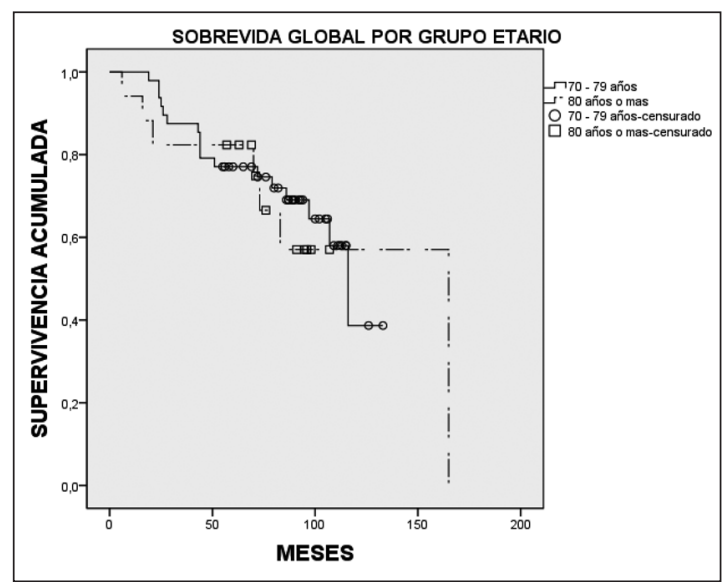

Figura 4. Sobrevida global según grupo etario. 
o para metástasis linfática axilar positiva comparada con negativa (Log Rank p: 0,13). La SG para las pacientes con subtipo tumoral luminal A comparada con el resto, demuestra tendencia a ser más prolongada, (Log Rank p: 0,08); sin lograr significancia estadística (Figura 5). La recidiva de $\mathrm{CM}$ ha sido local en 0 pacientes, 3 pacientes han desarrollado segundos primarios contra laterales y 11 enfermedad a distancia: Ósea 2, ósea y pleural 3 , ósea y hepática 2, peritoneal 2, hepática 2.

\section{Toxicidad por radioterapia}

Todas las pacientes completaron radioterapia sin interrupciones. El tratamiento tuvo una duración entre 35 y 44 días. La toxicidad aguda más frecuente fue eritema de la piel irradiada en grado leve $49 \%$ de los casos, moderada en $41 \%$ y severa en $10 \%$. Dos pacientes fallecieron por cáncer pulmonar de tipo escamoso, a los 72 y 86 meses de seguimiento.

\section{Discusión}

La incidencia de cáncer aumenta exponencialmente con la edad, con un riesgo acumulativo de 1 en 2 para hombres y 1 en 3 para mujeres a la edad de 80 años $^{22}$. El cáncer de mama en la mujer se comporta en forma similar aumentando su incidencia al avanzar la edad ${ }^{23}$. A pesar de la mayor frecuencia de CM en el AM no hay consenso acerca de los estudios de prevención con mamografía en las mujeres mayores de 70 años ${ }^{24}$. Tampoco se ha definido la terapia sistémica para este grupo etario, siendo escasos los estudios fase III ${ }^{8,25}$, por lo que las decisiones terapéuticas para el AM son basadas en indicaciones definidas para las pacientes más jóvenes, modificadas según la presencia de co-morbilidades, la valoración de la capacidad funcional y las expectativas de vida ${ }^{3,6,26}$.

Estudios previos han indicado que los AM con CM consultan en etapas clínicas más avanzadas, siendo pocas las pacientes diagnosticadas en etapas precoces o con tumores in situ (no infiltrantes) ${ }^{26}$. Los datos de CM del Ministerio de Salud de Chile el año 2009, para la población general, indican que se diagnosticó el CM en etapas I-II en $72 \%$ y etapa III en $28 \%{ }^{27,28}$. En nuestro estudio, $77 \%$ de las pacientes AM se diagnosticaron en etapas I-II y $23 \%$ en etapa III. Estos resultados difieren con los publicados previamente para los $\mathrm{AM}^{27,28}$ y se

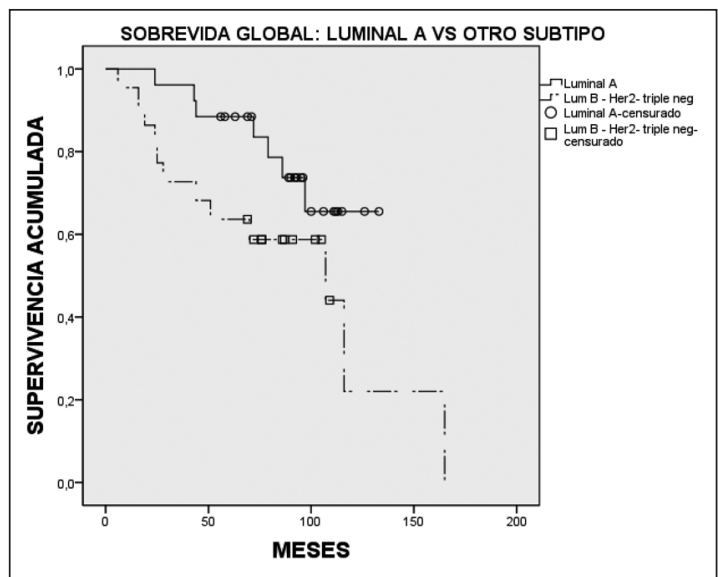

Figura 5. Sobrevida global según subtipo clínico patológico.

asemejan a la población general de Chile ${ }^{27,28}$. Las diferencias observadas en nuestro estudio pueden deberse a factores demográficos, socioeconómicos y a los criterios de inclusión utilizados para este estudio.

En nuestro reporte, las mujeres AM que fueron diagnosticadas en base a hallazgos en la mamografía preventiva y no por signos o síntomas, fueron diagnosticadas con cáncer en etapas más tempranas, y cursaron con mejor SG y SEE. Puede existir un sesgo de selección que explique el beneficio del tamizaje: mujeres que se realizan estudios prevención estarían en mejores condiciones funcionales $\mathrm{y}$ con mejores expectativas de vida.

A pesar de estos factores, parecería beneficioso continuar la prevención con mamografía en las mujeres AM.

La presencia de co-morbilidades es frecuente en AM, representado un riesgo competitivo en el análisis de mortalidad ${ }^{7}$. En nuestra población cerca de la mitad de las causas de fallecimiento fueron relacionadas al CM, remarcando la importancia del cáncer como causa de mortalidad en el AM. El número reducido de pacientes en nuestro trabajo no permite demostrar diferencias significativas en mortalidad para los AM con mayores niveles de co-morbilidad.

Los marcadores patológicos convencionales (RE, RP, HER2, GH) han demostrado que la determinación de subtipos clínico-patológicos, asimilando los subtipos moleculares descritos por Perou et $\mathrm{al}^{29}$, son de valor pronóstico ${ }^{12,13,14}$. Debido a la ausencia del valor de Ki67 en nuestras 
muestras, decidimos, en un análisis exploratorio, y como lo han hecho otros autores ${ }^{30}$, utilizar la diferenciación histológica $(\mathrm{GH})$ para catalogar a nuestros pacientes como luminales A o B. Así, las pacientes categorizadas luminal A tuvieron una mejor sobrevida, de acuerdo con lo publicado. Nuestros resultados plantean la factibilidad de implementar esta clasificación en nuestro medio dada su importancia pronóstica.

Desde el punto de vista inmuno-fenotípico la mayoría de los tumores en AM tienen un perfil endocrino-respondedor, con predominio de tumores de tipo luminal, con tendencia a cursar con mejor sobrevida que aquellos no luminales ${ }^{3,29}$. Esta caracterización se observa también en nuestro estudio, teniendo la mayoría de las pacientes un perfil endocrino-respondedor por lo que reciben terapia hormonal adyuvante tras la cirugía o como manejo inicial del $\mathrm{CM}^{31,32}$.

Todas las mujeres AM completaron la radioterapia sin interrupciones. La toxicidad durante la radiación no limitó su aplicación en este grupo etario. Los resultados en el control local fueron excelentes y similares a los observados en mujeres de menor edad. La naturaleza retrospectiva de nuestro estudio no permite evaluar de forma estandarizada la toxicidad a largo plazo de la radioterapia. El número reducido de pacientes analizados no permite evaluar con certeza la incidencia de neoplasias radio-inducidas, observamos, sin embargo, que dos pacientes desarrollaron cáncer pulmonar escamoso, lo que representa una tasa mayor a la esperada para la población general ${ }^{33}$.

\section{Conclusiones}

En nuestro estudio las mujeres AM con CM que realizaron prevención primaria con mamografía, tamizaje, tienen una mejor SG que las pacientes diagnosticadas por la hallazgos clínicos. La clasificación clínico-patológica del CM se correlaciona con la evolución clínica de las pacientes AM. EL CM fue la causa de muerte en más de $40 \%$ de las mujeres AM, lo que nos exige estudiar y desarrollar formas efectivas de tratamiento del CM para esta población, considerando la edad y la presencia de co-morbilidades. La radioterapia fue bien tolerada resultando en excelente control local, pero falta estudiar en forma sistemática sus efectos a largo plazo $^{34}$. Todas las pacientes recibieron y completaron todas sus sesiones de radioterapia sin interrupciones. Esto indica que no hubo toxicidad severa que obligara a suspender temporalmente la radioterapia.

\section{Referencias}

1. Censo de Población y Vivienda. Instituto Nacional de Estadística. 2002.

2. http://ccp.ucr.ac.cr/bvp/pdf/vejez/ageams.pdf (consultado el 15 de junio de 2013).

3. Kimmick G, Muss HB. Breast cancer in older patients. Semin Oncol 2004; 31: 234-48.

4. Departamento de Estadística e Información en Salud 2009. Ministerio de Salud. Gobierno de Chile. Disponible en: www.deis.cl. [Consultado el 10 de abril de 2012].

5. Yancik R, Ries LG, Yates JW. Breast cancer in aging women. A population-based study of contrasts in stage, surgery, and survival. Cancer 1989; 63: 976-81.

6. Yancik R, Wesley MN, Ries LA, Havlik RJ, Edwards BK, Yates JW, et al. Effect of age and comorbidity in postmenopausal breast cancer patients aged 55 years and older. JAMA 2001; 285: 885-92.

7. Satariano WA, Ragland DR. The effect of comorbidity on 3-year survival of women with primary breast cancer. Ann Intern Med 1994; 120: 104-10.

8. Hutchins LF, Unger JM, Crowley JJ, Coltman CA JR, Albain KS. Underrepresentation of patients 65 years of age or older in cancer-treatment trials. N Engl J Med 1999; 341: 2061-7.

9. http://www.cancerstaging.org/products/pasteditions. html (consultado el 15 de junio de 2013).

10. Elston CW, Ellis IO. Pathological prognostic factors in breast cancer. I. The value of histological grade in breast cancer: experience from a large study with long-term follow-up. Histopathology 1991; 19: 403-10.

11. Bloom HJG, Richardson WW. Histological grading and prognosis in breast cancer. A study of 1409 cases of which 359 have been followed for 15 years. Br J Cancer 1957; II: 359-77.

12. Allison KH, Kandalaft PL, Sitlani CM, Dintzis SM, Gown AM. Routine pathologic parameters can predict Oncotype DX recurrence scores in subsets of ER positive patients: who does not always need testing? Breast Cancer Res Treat 2012; 131: 413-24.

13. Flanagan MB, Dabbs DJ, Brufsky AM, Beriwal S, Bhargava R. Histopathologic variables predict Oncotype DX recurrence score. Mod Pathol 2008; 21: 1255-61.

14. Kamal AH, Loprinzi CL, Reynolds C, Dueck AC, Geiger $\mathrm{XJ}$, Ingle JN, et al. Breast medical oncologists' use of 
standard prognostic factors to predict a 21-gene recurrence score. Oncologist 2011; 16: 1359-66.

15. Edge SB. Breast. In: Edge SB, Byrd DR, Compton CC, et al., eds.: AJCC Cancer Staging Manual. 7th ed. New York, NY: Springer, 2010, pp 347-76.

16. http://www.nccn.org/professionals/physician_gls/pdf/ breast.pdf. (consultado el 15-06-13).

17. http://www.mastologia.cl/sitio/cnsss_guia.html. (consultado el 15-06-13).

18. Cox JD, Stetz J, Pajak TF. Toxicity criteria of the Radiation Therapy Oncology Group (RTOG) and the European Organization for Research and Treatment of Cancer (EORTC). Int J Radiat Oncol Biol Phys 1995; 31: 1341-46.

19. Hudis CA, Barlow WE, Costantino JP, Gray RJ, Pritchard KI, Chapman JA, et al. Proposal for standardized definitions for efficacy end points in adjuvant breast cancer trials: the STEEP system. J Clin Oncol 2007; 25: 2127-32.

20. http://www.minsal.cl/portal/url/item/7220fdc4341c44a9 e04001011f0113b9.pdf (consultado el 28 de octubre de 2013).

21. Morrow M, Harris JR, Schnitt SJ. Surgical margins in lumpectomy for breast cancer-bigger is not better. $\mathrm{N}$ Engl J Med 2012; 367: 79-82.

22. Jemal A, Siegel R, Xu J, Ward E. Cancer statistics, 2010. Ca Cancer J Clin 2010; 60: 277-300.

23. Balducci L, Extermann M. Management of cancer in the older person: a practical approach. Oncologist 2000; 5: 224-37.

24. Berry DA, Cronin KA, Plecritis SK, Fryback DG, Clarke L, Zelen M, et al. Effect of screning and adjuvant therapy on mortality from breast cancer. N Engl J Med 2005; 353: 1784-92.
25. McMurdo ME, Witham MD, Gillespie ND. Including older people in clinical research. BMJ 2005; 331: 10367.

26. Holmes CE, Muss HB. Diagnosis and treatment of breast cancer in the elderly. CA Cancer J Clin 2003; 53: 227-44.

27. http://www.ine.cl/canales/chile_estadistico/demografia _y_vitales/estadisticas_vitales/pdf/vitales_2010.pdf (consultado el 25 de junio de 2013).

28. Prieto M. Epidemiología del Cáncer de mama en Chile. Rev Med Clin Condes 2011; 22: 428-35.

29. Perou C, Sorlie T, Eisen B, Van De Rijnb M, Jeffrey, Rees $\mathrm{CA}$, et al. Molecular portraits of human breast tumours. Nature 2000; 406: 747-52.

30. Goldhirsch A, Wood WC, Coates AS, Gelber RD, Thürlimann B, Senn HJ; Panel members. Strategies for subtypes-dealing with the diversity of breast cancer: highlights of the St. Gallen International Expert Consensus on the Primary Therapy of Early Breast Cancer 2011. Ann Oncol 2011; 22: 1736-47.

31. Bernard-Marty C, Cardoso F, Piccart MJ. Facts and controversies in systemic treatment of metastatic breast cancer. Oncologist 2004; 9: 617-32.

32. Balducci L, Extermann M. Cancer and aging. An evolving panorama. Hematol Oncol Clin North Am 2000; 14: $1-16$.

33. Berrington De González A, Curtis RE, Gilbert E, Berg $\mathrm{CD}$, Smith SA, Stovall M, et al. Second solid cancers after radiotherapy for breast cancer in SEER cancer registries. Br J Cancer 2010; 102: 220-6.

34. Demirci S, Nam J, Hubbs JL, Nguyen T, Marks LB. Radiation-induced cardiac toxicity after therapy for breast cancer: interaction between treatment era and follow-up duration. Int J Radiat Oncol Biol Phys 2009; 73: 980-7. 